\title{
ESTRATEGIAS DE APRENDIZAJE COLABORATIVO \\ EN ESTUDIANTES DE NIVEL MEDIO SUPERIOR \\ EN LAS UNIDADES DE APRENDIZAJE \\ BIOLOGÍA Y FUNDAMENTOS DE GENÉTICA Y BIOTECNOLOGÍA
}

\section{COLLABORATIVE LEARNING STRATEGIES IN UPPER MIDDLE LEVEL STUDENTS IN THE BIOLOGY AND FUNDAMENTALS OF GENETICS AND BIOTECHNOLOGY LEARNING UNITS}

\author{
Irma Carolina Reyna Rodríguez \\ Universidad Autónoma de Nuevo León, México \\ mvz.carolreyna@gmail.com \\ https://orcid.org/0000-0003-3925-297X \\ Fernando Javier Gómez Triana \\ Universidad Autónoma de Nuevo León, México \\ fernando.gomez.21@hotmail.com \\ https://orcid.org/0000-0003-2652-8949
}

\begin{abstract}
RESUMEN
La investigación tuvo el propósito de examinar la aplicación de actividades lúdicas y prácticas de laboratorio en el nivel de enseñanza medio superior, en las unidades de aprendizaje Biología y Fundamentos de Genética y Biotecnología. Se intentó detectar el beneficio de tales actividades y prácticas en función del aprovechamiento de los alumnos y de la reducción del número de estudiantes con segunda oportunidad. Se utilizó un diseño cuasi-experimental con una muestra total de 387 estudiantes. Los resultados mostraron un aumento en el índice de aprobación en los grupos donde se realizaron actividades lúdicas y prácticas de laboratorio. La experiencia recomienda implementar tales actividades y prácticas para un mejor aprovechamiento escolar.
\end{abstract}

Palabras clave: aprendizaje colaborativo, actividades lúdicas, prácticas de laboratorio, aprovechamiento escolar

\section{ABSTRACT}

The research was intended to examine the application of games activities and laboratory practices at the upper secondary level, in the Biology and Fundamentals of Genetics and Biotechnology learning units. The purpose was to detect the benefit of such activities and practices based on student achievement and the reduction in the number of students with a second chance. A 
quasi-experimental design was used with a total sample of 387 students. The results showed an increase in the approval rate in the groups where game activities and laboratory practices were carried out. Experience recommends implementing such activities and practices for better academic achievement.

Keywords: collaborative learning, game activities, laboratory practices, school achievement

\section{Introducción}

A través de la historia, el aprendizaje ha sido estudiado desde distintos puntos de vista, abarcando los procesos involucrados a din de descubrir en qué consiste y encontrar los elementos intervinientes y el y modo en que se manifiesta y optimiza.

Sin embargo, únicamente se puede hablar de aprendizaje cuando el cambio que se produce es duradero, para lo cual la práctica resulta imprescindible (Díaz Mosquera, 2012).

Algunas teorías mencionan que el aprendizaje colaborativo es direccionado desde una perspectiva de desarrollo, como un proceso biológico y/o cultural que ocurre con los años. En este espectro de definiciones, también se incluye al aprendizaje que es logrado por la adquisición de experticia dentro de una comunidad profesional. (Collazos Ordoñez, Guerrero y Vergara, 2001, p. 2)

Sin embargo, no siempre se logran estos resultados. Springer, Stanne y Donovan (1999) realizaron un estudio de revisión centrado en la comparación de investigaciones sobre los efectos del trabajo de pequeños grupos en el aprendizaje cooperativo o colaborativo. Los resultados de este estudio no presentan cambios relevantes en el rendimiento de los estudiantes que trabajaron en pequeños grupos en aprendizaje coope- rativo, aprendizaje colaborativo o una mezcla de ambos. Concluyeron que cualquier método que mantenga a los estudiantes activos en el aprendizaje puede tener efectos positivos, con lo cual coinciden también Goikoetxea y Pascual (2002).

Por otra parte, se ha encontrado en estudios recientes que la aplicación de actividades lúdicas y de prácticas de laboratorio que se implementan para beneficiar al aprendizaje colaborativo es acertada para lograr los objetivos de la investigación. Así mismo, se encontró que es eficaz su aplicación incluso en el nivel superior. Tal es el caso de la Universidad de Entre Ríos, Argentina, donde Clérici (2012) estudió el rol del juego con un grupo de estudiantes de diferentes áreas como anatomía y fisiología, ciencias económicas, lingüística y comunicación humana, entre los periodos 2008-2011. Los resultados mostraron que la actividad lúdica constituye una herramienta valiosa para mejorar el rendimiento en el aprendizaje.

En lo que concierne a las prácticas de laboratorio, los estudiantes son beneficiados al desarrollar habilidades en el manejo instrumental en las prácticas, fomentando autonomía e interés por el área de investigación, aunados a una disciplina de trabajo organizado requerida en cualquier actividad (Severiche Sierra y Acevedo Barrios, 2013). 
El aporte de las prácticas de laboratorio enriquece el interés por aprender, por participar y relacionarse con las ciencias biológicas de manera más tangible para el estudiante. García Barros, Martínez Losada y Mondelo Alonso (1995) describen que, si se pretende promover el aprendizaje significativo de conceptos, junto al acercamiento del alumno a los procesos de la ciencia, resulta imprescindible que la formación docente haga especial énfasis en el análisis de contenidos implicados en diferentes tipos de actividades prácticas. Dicho análisis permite a los docentes aplicar sus conocimientos previos sobre contenidos conceptuales, procedimentales y actitudinales, vistos en las materias de carácter general, a los temas específicos de las ciencias de la naturaleza.

La importancia de realizar este proyecto radica en que, con la implementación de las actividades lúdicas y las prácticas de laboratorio, no solo se busca lograr un resultado eficaz por parte del estudiante al finalizar el curso, cuya relación busca determinar el presente estudio, sino también fomentar la participación del grupo, motivar al estudiante, despertar su interés por el estudio de la vida y los avances tecnológicos en la genética, y que conozcan que es algo de lo que ellos pueden ser partícipes en un futuro para quienes muestren interés en carreras encaminadas a dichas unidades de aprendizaje, aunque la medición de estas variables no se incluyeron en el proyecto.

Agudelo G. y García G. (2010) señalan la importancia de trabajar con estrategias que favorezcan las prácticas pedagógicas, en donde la experiencia fundamente un aprendizaje significativo en el estudiante.

Se espera que con el uso de las activi- dades lúdicas y las prácticas de laboratorio se pueda no solo obtener un buen resultado en la calificación del estudiante y un aprendizaje significativo, sino que se logre despertar el interés por conocer otras dinámicas de trabajo colaborativo que pueden ser divertidas e interesantes; así mismo, que facilite su comprensión de los contenidos abordados y que modifique su perspectiva sobre las unidades de aprendizaje como materias tediosas.

\section{¿Qué es el aprendizaje?}

A través de la historia en el ámbito educativo, se han estudiado diversas formas de enseñar y aprender. En términos generales, la enseñanza se entiende como la transmisión de conocimientos y el aprendizaje como la adquisición de conocimientos. Pero en términos profesionales, ¿cómo podemos definir qué es aprender?

Desde hace dos décadas se han manifestado dos tipos de visiones en la definición de aprendizaje. En la última década se enfatiza que el aprendizaje es construcción activa de conocimiento, no recepción pasiva de información. La valoración del aprendizaje es considerada cualitativamente y se presta atención a qué tipo de estructuras de conocimiento construyen los estudiantes y cómo las generan.

\section{El aprendizaje colaborativo}

El aprendizaje colaborativo no es innovador. Para Lara Ros (2001), los primeros estudios datan de 1938 con Thorndike y de 1949 con Deutsch. Luego, nuevamente en la década de los 70 , el aprendizaje cooperativo retoma su interés en el área educativa.

Sin embargo, en el trabajo colaborativo los grupos no constituyen el ente fundamental por el simple hecho 
de acomodar grupos en un aula y advertirles que son un grupo que deben colaborar o cooperar. Al formar grupos de trabajo colaborativo, es primordial supervisar que los elementos básicos estén estructurados de manera clara en cada sesión de trabajo. Sólo así, se puede lograr que se involucre el esfuerzo grupal, ligado a la colaboración y al resultado del mismo (Maldonado Pérez, 2008).

Este aprendizaje no se da naturalmente al exponer una instrucción o alguna tarea a un grupo de estudiantes, sino que es necesario tener como base una intención que, por medio del trabajo en equipo, propicie el aprendizaje esperado. Como resultado, esta experiencia en grupo debe manifestar un aprendizaje en particular para cada individuo. Sin duda, la interacción en la construcción del conocimiento promueve mejores y mayores resultados a diferencia del trabajo individual (Lillo Zúñiga, 2013).

El aprendizaje colaborativo solo puede darse en un ambiente empático en donde cada individuo sienta la libertad de poder expresarse y ser partícipe en la acción del trabajo grupal.

Otras actividades que apoyan la estrategia del aprendizaje colaborativo es el uso de las actividades lúdicas, en donde por medio del juego se puede dar la adquisición del conocimiento de algún contexto o tema específico de manera divertida y con la participación de todo el grupo.

Por su parte, las prácticas de laboratorio son actividades que hacen más atractivas y dinámicas las clases, además de promover el gusto por la investigación y la curiosidad por aprender.

\section{Actividades lúdicas}

Chacón (2008) menciona que los juegos requieren de la comunicación para provocar y activar los mecanismos de aprendizaje. Dentro del aula, el grupo se va envolviendo en un ambiente lúdico el cual genera que cada estudiante desarrolle sus propias estrategias de aprendizaje.

Por medio del juego, los docentes dejan de ser el centro de la clase, pasando a ser solo acompañantes en el proceso de aprendizaje. Sin duda la participación de todo el grupo es importante, tanto para amenizar el ambiente del aula, como para el logro de los objetivos del uso de dichas actividades. Además, se necesita estimar tiempo para planear la actividad a conciencia y no afectar la calidad del trabajo. El juego debe ser atractivo y contar con un grado de competición para estar a la par con los contenidos a manejar.

Las actividades mencionadas deben contribuir a promover el interés y la curiosidad por aprender en los estudiantes. Es por eso que otras actividades que se consideran como apoyo del presente proyecto dentro de las ciencias biológicas son las prácticas de laboratorio, las cuales ayudan a ser tangibles los contextos vistos en la unidad.

\section{Prácticas de laboratorio}

Las prácticas de laboratorio han pasado a ocupar un papel central en la didáctica de las ciencias experimentales y su importancia ha llegado a ser parte fundamental en la estimulación del aprendizaje. Estas son indispensables para aprender ciencias y precisan también que el alumno sepa qué es lo que está haciendo (Bastida de la Calle, Ramos Fernández y Soto López, 1990).

No obstante, una desventaja del uso de las prácticas de laboratorio es que el factor tiempo influye en su eficacia.

López Rúa y Tamayo Alzate (2012) afirman que las actividades de laboratorio 
mayormente se comparan con una receta, siguiendo pasos que llevarán a una conclusión prevista. Dentro de los obstáculos que se pueden presentar en los trabajos prácticos, se encuentran la falta de materiales y de espacios adecuados, las limitaciones de tiempo, el tamaño muy grande de los grupos, y la falta de motivación y disposición de los educandos y de algunos docentes.

Si la actividad cuenta con los elementos necesarios, el trabajo de laboratorio favorece y promueve el aprendizaje de las ciencias. Esto le brinda al estudiante el poder cuestionar sus saberes y confrontarlos con la realidad de manera tangible, entretenida y fomentando el interés por las ciencias experimentales, como lo son la biología, la química y la genética.

\section{Metodología}

Dentro de un enfoque cuantitativo, la investigación adoptó un diseño cuasi-experimental, que procuró predecir un valor estimado que tendrá un grupo de estudiantes en una variable como resultado de una intervención (Hernández Sampieri, Fernández Collado y Baptista Lucio, 1998). En este caso, como resultado de la aplicación de actividades lúdicas y prácticas de laboratorio. Se utilizó como muestra de estudio un grupo de estudiantes de nivel medio superior en la modalidad de bachillerato general, en el periodo de enero a junio de 2018. Se eligieron seis grupos de segundo semestre de la unidad de aprendizaje de Biología y dos grupos de cuarto semestre de la unidad de aprendizaje de Fundamentos de Genética y Biotecnología.

La muestra fue dividida en dos grupos poblacionales. El primero estuvo conformado por tres grupos de segundo semestre y uno de cuarto, a los que se intervino con estrategias de actividades lú- dicas y prácticas de laboratorio, integrado por un total de 204 estudiantes bajo el nombre de "Grupo A". Al segundo grupo de la muestra, se asignaron otros tres grupos de segundo semestre y otro grupo de cuarto semestre, a quienes no se aplicaron dichas actividades a lo largo del semestre. Este grupo de control estuvo conformado por 183 de estudiantes a quienes se les asignó por nombre "Grupo B". De esta manera, la muestra final estuvo conformada por 387 estudiantes.

Como instrumento, se utilizaron juegos de destreza tradicionales acondicionados a los temas vistos en cada unidad de aprendizaje, tales como lotería, memorama, rompecabezas, adivina quién soy, el ahorcado, jenga, quiz pastelazo, crucigrama y quiz interactivo digital.

Por otra parte, dentro de las prácticas de laboratorio, se realizó la identificación de las fases de la mitosis con las capas de una cebolla en el microscopio, la identificación de agentes etiológicos en el microscopio y la disección del corazón, miembros posteriores y aparato reproductor femenino de cerdo y vaca para la identificación de estructuras y funciones. La aplicación de las actividades lúdicas se realizó una semana previa a iniciar los exámenes del primer parcial. Lo mismo sucedió antes del segundo examen parcial, en tanto las prácticas de laboratorio se realizaron antes de la semana de exámenes globales en el laboratorio de biología.

\section{Resultados}

De acuerdo con el reporte de evaluaciones basado en la plataforma del Sistema Integral para la Administración de los Servicios Educativos (SIASE), los resultados de aprobación y desaprobación para cada grupo se grafican en la Figura 1. 


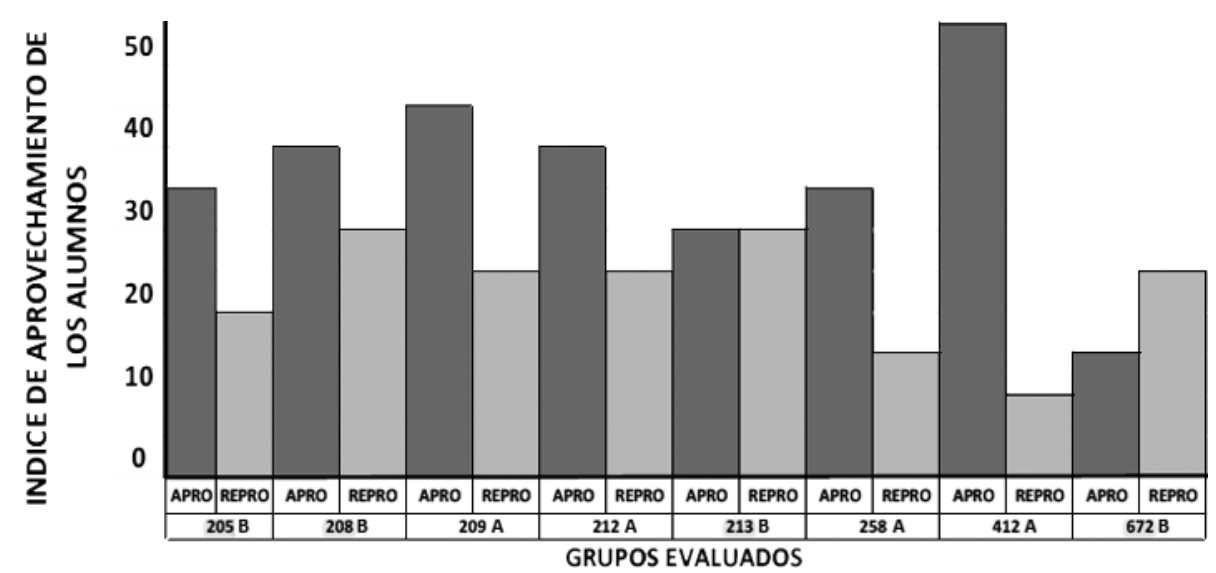

Figura 1. Representación gráfica del índice de aprovechamiento de los 387 estudiantes evaluados por grupo.

Con base en la evaluación sumativa de la plataforma del SIASE, que consta del porcentaje de portafolio y de exámenes de los estudiantes, se puede ver el índice de aprobación en color azul y el índice de reprobación en color naranja por grupo. Esto marca mayor aumento en el aprovechamiento de los estudiantes que realizaron las prácticas.

Dentro del Grupo A, 145 estudiantes de los 204 (71\%) implicados en las estrategias aprobaron las unidades, en comparación con el Grupo B, con 101 estudiantes aprobados de 183 (55\%) que no participaron en las estrategias.

A pesar de ser menor el número de estudiantes que no participaron de las estrategias, el índice de reprobados del Grupo B $(n=82)$ fue mucho mayor que el del Grupo A $(n=59)$.

Una prueba $t$ permitió observar que las medias de calificación de los grupos fueron significativamente diferentes $\left(t_{(348.976)}=10.160, p=.000, \eta^{2}=.215\right)$. El grupo A obtuvo una media significativamente mayor $(M=70.72, D E=$ $0.33)$ que el grupo $\mathrm{B}(M=65.05, D E$ $=0.44)$.

\section{Discusión}

De acuerdo con los resultados obtenidos en la evaluación sumativa del Grupo A y el Grupo B, podemos concluir que nuestro objetivo principal ha sido comprobado de manera eficaz, reflejándose en el índice de aprobación de los grupos de estudiantes que participaron en la aplicación de las actividades lúdicas y en las prácticas de laboratorio mediante el aprendizaje colaborativo.

Sin duda, el anexar dentro del curso actividades lúdicas y prácticas de laboratorio brindaron al estudiante un ambiente colaborativo y constructivo, haciendo menos tediosos los contenidos de las asignaturas de Biología y Fundamentos de Genética y sirviendo como un repaso dinámico para la adquisición de los conceptos previos a presentar sus exámenes.

Por lo tanto, esta aproximación puede ser considerada como una buena estrategia que apoya no solo en el índice de aprobación en las asignaturas observadas, sino en cualquier unidad de aprendizaje en donde se pueda desarrollar el trabajo en equipo. 
Hay nuevos retos que se avecinan en el próximo periodo escolar, debido al cambio del modelo educativo en la educación media superior. Es importante considerar el uso de este tipo de estrategias, ya que al verse modificado el tipo de evaluación sumativa, en donde se les dará un mayor porcentaje a los exámenes $(60 \%)$ y uno menor al portafolio de trabajo $(40 \%)$, la interacción y la colaboración serán necesarias para el logro de las competencias, así como el apoyo al fortalecimiento de las habilidades socioemocionales que en este nuevo modelo educativo se deben impulsar.

Será oportuno que los docentes adquieran las herramientas necesarias y se capaciten continuamente para preparar a los estudiantes en las nuevas asignaturas fusionadas y que logren obtener un mayor aprovechamiento en su rendimiento académico.

En suma, los resultados muestran la conveniencia de aplicar las actividades lúdicas y prácticas de laboratorio en las unidades de aprendizaje que se lleven a futuro en todas las dependencias.

\section{Referencias}

Agudelo G., J. D. y García G., G. (2010). Aprendizaje significativo a partir de prácticas de laboratorio de precisión. Latin-American Journal of Physics Education, 4(1), 149-152.

Bastida de la Calle, M. F., Ramos Fernández, F. y Soto López, J. (1990). Prácticas de laboratorio: ¿una inversión poco rentable? Revista Investigación en la Escuela, (11), 77-91. https://doi .org/10.12795/IE.1990.i11.08

Chacón, P. (2008). El juego didáctico como estrategia de enseñanza y aprendizaje ¿Cómo crearlo en el aula? Nueva Aula Abierta, 5(16). Recuperado de http://www.e-historia.cl/ cursosudla/13EDU413/lecturas/06\%20\%20 E1\%20Juego\%20Didactico\%20Como\%20 Estrategia\%20de \%20Ense $\% \mathrm{C} 3 \% \mathrm{~B} 1$ anza $\% 20$ y\%20Aprendizaje.pdf
Clérici, C. (2012). El juego como estrategia de enseñanza y aprendizaje en el nivel superior. Diálogos Pedagógicos, 10(19), 136-140.

Collazos Ordoñez, C. A., Guerrero, L. y Vergara, A. (2001, noviembre). Aprendizaje colaborativo: un cambio en el rol del profesor. En Actas del Tercer Congreso de Educación Superior en Computación. Punta Arenas, Chile.

Díaz Mosquera, E. (2012). Estilos de aprendizaje. Eidos, 5, 5-11. https://doi.org/10.29019/eidos .$v 0 \mathrm{i} 5.88$

García Barros, S., Martínez Losada, C. y Mondelo Alonso, M. (1995). El trabajo práctico: una intervención para la formación de profesores. Enseñanza de las Ciencias, 13(2), 203-209.

Goikoetxea, E. y Pascual, G. (2002). Aprendizaje cooperativo: bases teóricas y hallazgos empíricos que explican su eficacia. Educación XX1, 5, 227-247. https://doi.org/10.5944/educxx1 .5 .1 .392

Hernández Sampieri, R., Fernández Collado, C. y Baptista Lucio, P. (1998). Metodología de la investigación. México: McGraw-Hill.

Lara Ros, S. (2001). Una estrategia eficaz para fomentar la cooperación. Estudios sobre Educación, 1, 99-110.

Lillo Zúñiga, F. G. (2013). Aprendizaje colaborativo en la formación universitaria de pregrado. Revista de Psicología, 2(4), 109-142.

López Rúa, A. M. y Tamayo Alzate, Ó. E. (2012). Las prácticas de laboratorio en la enseñanza de las ciencias naturales. Revista Latinoamericana de Estudios Educativos (Colombia), 8(1), 145-166.

Maldonado Pérez, M. (2008). Aprendizaje basado en proyectos colaborativos. Una experiencia en educación superior. Laurus, 14(28), 158-180.

Severiche Sierra, C. A. y Acevedo Barrios, R. L. (2015). Las prácticas de laboratorio en las ciencias ambientales. Revista Virtual Universidad Católica del Norte, 40, 191-203. Recuperado de https://www.redalyc.org/articulo .oa?id=194229200014

Springer, L., Stanne, M. E. y Donovan, S. S. (1999). Effects of small-group learning on undergraduates in science, mathematics, engineering, and technology: A meta-analysis. Review of Educational Research, 69, 21-51. https://doi .org/10.3102/00346543069001021

Recibido: 3 de abril de 2019 Revisado: 13 de mayo de 2019 Aceptado: 3 de junio de 2019 